\title{
Sentinel surveillance of Lymphatic filariasis, Schistosomiasis, Soil transmitted helminths and Malaria in rural southern Malawi
}

\author{
Kelias Msyamboza ${ }^{1}$, Bagrey Nowira ${ }^{2}$, Richard Banda ${ }^{1}$,Square \\ 1World Health Organisation, Malawi Country Office, Lilongwe, Malawi \\ 2Community Health Department, College of Medicine, Blantyre, Malawi \\ 3LF Control Programme, Ministry of Health, Lilongwe, Malawi \\ 4Child and Reproductive Health Group, Liverpool School of Tropical Medicine, UK \\ 5EmmaKinderziekenhuis, Academic Medical Centre, University of Amsterdam, The \\ Netherlands \\ 6Department of Community Paediatrics, Royal Liverpool Children's Hospital NHS \\ Trust, Alder Hey, UK \\ Correspondence: msyambozak@mw.afro.who.int
}

\begin{abstract}
Background: Baseline prevalence and knowledge, attitude and perception (KAP) survey is a prerequisite for mass drug administration for the control of Lymphatic filariasis (LF) and other neglected tropical diseases.

Methods: In preparation for the first mass drug administration for LF elimination, a baseline survey was conducted in six sentinel sites in the southern Malawi, amongst participants aged five years or more. A standard questionnaire was used to obtain data on socio-demographic factors, ownership and use of bed nets, previous ingestion of ivermectin and KAP toward hydrocele and lymphoedema. Finger prick blood samples were collected from 22:00 to 01:00 hours for LF microscopy, malaria and haemoglobin examination. Stool and urine samples were collected for internal helminths and schistosomiasis respectively.
\end{abstract}

Results: A total of 1, 903 participants were enrolled. Knowledge on the cause of hydrocele and lymphoedema was low in all the sentinel sites $(16 \%$ $42 \%, 10 \%-24 \%$ (respectively). Sexual intercourse with a menstruating woman, bad weather and HIV/AIDS were perceived causes of hydrocele. Microfilaraemia prevalence was $1.5 \%$ and varied little between sentinel sites $(1.0 \%-2.1 \%)$. Childhood urinary schistosomiasis was common in Phalombe $(94.9 \%)$ and Blantyre $(26.9 \%)$.

Conclusion Integrated approach and understanding of the community KAP is vital or successful implementation of LF elimination programme.

\section{Introduction}

Lymphatic filariasis (LF) is the second most common vectorborne parasitic disease after malaria ${ }^{1}$ and is the second commonest cause of long-term disability aftermental illness ${ }^{2,3}$. It is endemic in 83 countries, with more than 1.2 billion people at risk of infection. Some 120 million people are affected worldwide of whom about 40 million are incapacitated and disfigured by the disease ${ }^{4}$. The common

manifestations of LF are hydrocele, lymphoedema and elephantiasis. One-third of people infected with LF live in India, a third in Africa and the remainder in Latin America, the Pacific Islands, Papua New Guinea and South-East Asia ${ }^{1}$. In 1997, a World Health Assembly resolution called for the elimination of LF. The strategy for LF elimination is focused on interrupting the transmission of the parasite through the use of mass drug administration (MDA), once annually for a period of four to six years ${ }^{5}$. In addition, since LF is transmitted by mosquitoes, malaria vector control interventions such as insecticide treated nets (ITNs) and in-door residual spray (IRS) are likely to play a significant role on LF elimination. Mapping in 2003 showed LF was endemic in all the districts of Malawi except Chitipa $^{6}$. It is co-endemic with Onchocerciasis (Oncho) in six districts namely; Mwanza/Neno, Chikwawa, Blantyre, Thyolo, Chiradzulu, and Mulanje/Phalombe ${ }^{7-9}$. It is also co-endemic with soil transmitted helminths (STH) and
Schistosomiasis $(\mathrm{SCH})$ in these districts ${ }^{10-13}$.

Based on the findings of LF mapping, Malawi qualified for free donation of Mectizan ${ }^{\circledR}$ (Ivermectin) and Albendazole from Merck \& Co and GSK respectively through the Global Programme to Eliminate Lymphatic filariasis (GPELF). The Action Plan for elimination of LF was integrated with control of Onchocerciasis, SCH, STH and malaria. In the first year, integrated MDA was implemented in six districts selected because the African Programme for Onchocerciasis Control (APOC) had been operating in these since 1997 and they had well established community structures.

The aim of the present survey was to obtain baseline data on the prevalence of microfilaria, $\mathrm{STH}, \mathrm{SCH}$, anaemia and malaria parasitaemia and knowledge, attitude and perceptions of the community toward LF. The data was used for development community specific social mobilisation messages and strategies and monitoring and evaluation of the integrated mass drug administration for the control of LF, Oncho and intestinal worms. The survey was conducted in August 2008.

\section{Materials and Methods}

Six sentinel sites were selected in the six Oncho districts in the southern region using WHO guidelines ${ }^{14}$.

Individuals aged over four years were eligible. Recruitment was conducted from 18:00 hours starting with obtaining informed written consent and demographic data.

Finger prick blood samples for microfilaria were collected between 22.00 and 01.00 hours. Blood samples were collected into heparinised tubes from consenting participants by trained technicians and transported for later processing at Queen Elizabeth Central Hospital, Blantyre, or the Community Health Services Unit, Lilongwe.

In children aged 5 to 14 years, in addition to LF microscopy, a finger prick blood sample was also used for haemoglobin and malaria thick smear examination. Urine and stool samples were collected for examination for schistosomiasis and soil transmitted helminths respectively.

For LF microfilaria microscopy, a Sedgewick counting chamber was used.

Haemoglobin was measured by HemoCue ${ }^{\circledR}$ (Hb 201+ Angelholm Sweden). Malaria slides were prepared with Field's A and B stain and air dried. A malaria slide was considered negative if no asexual parasites were observed in 100 fields. Urine was centrifuged and examined for schistosoma and a wet stool preparation was examined for helminths.

Knowledge, attitudes and perceptions of the community 
toward LF was obtained from adults aged 15 years or more through individual interviews using a semi-structured

questionnaire.

Data was entered in Microsoft excelß, Epi-Info 2004 (Centres for Disease Control and Prevention, Atlanta, Ga) and exported to SPSS (Chicago IL) for analysis. Participants under 14 years were considered children and older participants as adults. Anaemia was defined as haemoglobin $<11.0 \mathrm{~g} / \mathrm{dl}$.

Ethical clearance was granted by the National Health Sciences Research Committee.

\section{Results}

A total of 1, 903 participants were enrolled with sample sizes in sentinel sites ranging from 126 in (Blantyre) to 477 in (Thyolo), (table 1). The proportion of males and females were similar. Bed net ownership varied from 11\% in Thyolo to $37 \%$ in Blantyre, with an average of $17.4 \%$ of respondents sleeping under a bed net in the previous night. common manifestation of LF (hydrocele, lymphoedema, elephantiasis) was poor in contrast to that for manifestations of onchocerciosis (itching, skin nodules, skin thickening) which previously had been reported to be high (94\%) in these six districts. ${ }^{15}$

The lack of specific local names emphasises the importance of developing and intensifying community specific information, education and communication messages in order for integrated MDA to be successful. Lack of knowledge, or misconceptions about LF, have been shown previously to be important factors associated with poor compliance in MDA programmes. ${ }^{1,16,17}$

\section{Table 1: Demogtaphic characteristics of participants}

\begin{tabular}{|l|llllllll|}
\hline $\begin{array}{l}\text { Sentinel } \\
\text { site }\end{array}$ & $N$ & \multicolumn{9}{|c}{ Gender\% } & Age $\%$ & \multicolumn{3}{c|}{ Owns bed net\% } & ITN previous night \% \\
& & & & & & & \\
Any & ITN & & \\
\hline Blantyre & 126 & 60.3 & 39.7 & 43.0 & 57.0 & 37.3 & 29.3 & 18.4 \\
\hline Chikwawa & 305 & 50.3 & 49.4 & 64.2 & 35.8 & 30.8 & 29.9 & 29.2 \\
\hline Chiradzulu & 428 & 43.5 & 56.5 & 50.3 & 49.7 & 21.0 & 17.5 & 14.7 \\
\hline Mwanza & 303 & 44.6 & 55.4 & 43.4 & 56.4 & 34.0 & 25.1 & 23.8 \\
\hline Phalombe & 264 & 48.9 & 51.1 & 49.2 & 50.8 & 20.4 & 18.8 & 16.2 \\
\hline Thyolo & 477 & 46.8 & 53.2 & 46.7 & 53.3 & 10.9 & 9.2 & 8.2 \\
\hline All & 1903 & 47.5 & 52.5 & 49.7 & 50.3 & 23.1 & 19.3 & 17.4 \\
\hline
\end{tabular}

\section{Discussion}

Prevalence estimates of lymphatic filiariasis were lower than expected ( $<3 \%$ in all the sentinel sites). Surveys using a rapid diagnostic antigen test have reported higher prevalence with an estimate of $30 \%$ in Chikwawa compared to $2.1 \%$

prevalence for the same location in the present survey ${ }^{6}$. Use of Ivermectin in these areas as part of the Onchocerciasis Control Programme may explain the low prevalence. The observation that the mean prevalence was $1.5 \%$ for all survey sites indicates by definition that this area is endemic for LF and that MDA is justifiable.

The required sample size for sentinel site $\mathrm{LF}$ testing recommended by WHO is 500 participants. The lower participation in this survey is a limitation which could influence prevalence estimates. Low participation in night surveillance surveys can be problematic. The present survey was hindered by initiation ceremonies for boys (manganje) in the Blantyre area which reduced turnout as manganje was a highly valued traditional ceremony in this community. There were also fears that the survey workers might be practising 'satanism'. In this view, the routine night travel made survey teams vulnerable to attack and armed police officers were required to escort some survey teams. Poor weather; heavy rains and cold temperatures also influenced

low participation in this survey. Knowledge of the
Anaemia was common in children surveyed with the highest prevalence in Chikwawa where malaria is endemic. Use of bed nets on the previous night was less than $40 \%$ in all sites. Malaria prevalence was highest in Masanjala (19.5\%) where bed net usage on the previous night was $18.4 \%$. S.haematobium was endemic in all sites with almost all children infected who were surveyed in Phalombe (94.9\%).

Urinary schistosomiasis in Southern Malawi is a major public health problem and in a survey of 779 adolescent girls in the wet season of 2006 in Chikwawa, $28.4 \%$ reported macroscopic haematuria (18). In contrast, soil-transmitted helminths were less common which is consistent with previous findings in children from this area who had $<5 \%$ prevalence $^{19}$.

In conclusion operational limitations to night survey activities may be influenced by cultural factors and therefore timing to avoid ceremonial periods is important. Endemic helminth infections in this area of southern Malawi justify the provision of MDA. Improving access to and use of bed nets must be achieved to reduce LF and malaria transmission. Improving the communities' understanding, knowledge and perceptions of the complications of filariasis, as well as other endemic parasitic diseases, is vital for developing community specific social mobilisation messages and strategies for successful implementation of a programme for elimination 
of lymphatic filariasis.

\section{Acknowledgements}

We are grateful to the District Health Management Teams, Surveyors and chiefs for support they rendered to the survey. We are also grateful to WHO Malawi Country Office and WHO AFRO for financial and technical support.

\section{References}

1. Wynd S, Melrose WD, Durrheim DN, Carron J, Gyapong M (2007). Understanding the community impact of lymphatic filariasis: a review of the sociocultural literature. Bull World Health Org 85: 6 [cited 200810-21], pp. 493-498

2. Ottesen EA, Duke BOL, Karam M, Behbehani K (1997). Strategies and tools for the control/elimination of lymphatic filariasis. Bull World Health Org 75: 491-503.

3. World Health Organisation (1999). Building partnerships for lymphatic filariasis - strategic plan. Geneva: WHO

4. Ottesen EA, Hooper PJ, Bradley M, Biswas G (2008). The Global Programme to Eliminate Lymphatic Filariasis: Health Impact after 8 years. PLoS Neglected Tropical Diseases 2(10): e317

5. World Health Organisation (2002). Lymphatic filariasis: the disease and its control. Fifth report of the WHO expert committee on filariasis. Geneva: WHO

6. Ngwira BM, Tambala P, Perez AM, Bowie C, Molyneux DH (2007). The geographical distribution of lymphatic filariasis infection in Malawi. Filaria Journal 6:12 doi:10.1186/1475-2883-6-12

7. African Programme for Onchocerciasis Control (2006). Rapid epidemiological mapping of Onchocerciasis in Malawi. Geneva: WHO

8. Burnham GM. Onchocerciasis in Malawi (1991). 1. Prevalence, intensetity and geographical distribution of Onchocerca volvulus infection in Thyolo highlands. Trans Roy Soc Trop Med Hyg 85: 493-6

9. Courtright P, Johnston K, Chitsulo L (1995). A new focus of
Onchocerciasis in Mwanza district, Malawi. Trans Roy Soc Trop MedHyg 89: 34-6

10. National Schistosomiasis Control Programme, Malawi (2000). Ten year plan of action 1991-2000. Ministry of Health and Population, Lilongwe

11. Schistosomiasis Control Programme - Community Health Surveillance Unit 1997-2001 (2001). Lakeshore Schistosomiasis Control Project. Ministry of Health and Population, Lilongwe

12. Randall AE, Perez MA, Floyd S, Black GF, Crampin AC, et al. (2002). Patterns of helminths infection and relationship to BCG vaccination in Karonga District Northern Malawi. Trans $R$ Soc Trop Med Hyg 96:29-33.

13. Bowie C, Purcell B, Shaba B, Makaula P, Perez M (2004). A national survey of the prevalence of schistosomiasis and soil transmitted helminths in Malawi. BMC Infect Dis. 4: 49. [PubMed]

14. World Health Organisation (2005). Monitoring and epidemiological assessment of the programme to eliminate lymphatic filariasis at implementation unit level. Geneva: WHO

15. Johnston K, Courtright P, Burnham G (1994). Knowledge and attitude toward Onchocerciasis in Thyolo highlands of Malawi. Trop Med and Parasitol 45(4): 341-343

16. Talbot JT, Vial A, Direny A, de Rochars MB, Addiss D, et al. (2008). Predictors of compliance in mass drug administration for the treatment and prevention of lymphatic filariasis in Leogane, Haiti. Am J Trop Med Hyg 78:283-288

17. Kyelem D, Biswas G, Bockarie MJ, Bradley MH, et al. (2008). Determinants of Success in National Programs to Eliminate Lymphatic Filariasis: A Perspective Identifying Essential Elements and Research Needs. Am J Trop Med Hyg 79: 480-484

18. Kalanda GTC. Seasonal Patterns of Malaria and its Health Related Consequences Among Adolescent Females in Rural Malawi. PhD Thesis, University of Liverpool, 2008.

19. Calis JCJ, Phiri KS, Faragher B, Brabin BJ, Cuevas LC, De Haan RJ, Phiri AI et al (2008). Severe anaemia in Malawian children. New England Journal Medicine 358:888-899.

\section{Minority Health - Healthy Living Tips for Kids}

Make sure your children receive regular check-ups and have their shots on schedule. Teach your children good health habits like regularly washing their hands, good oral hygiene (brushing and flossing their teeth). Help children avoid unintentional injuries. Have them wear a helmet or other protective equipment if they are riding a bike, skateboarding, or rollerblading. All small children should be in a child safety seat or seat belt when riding in a car. Also make seat belts a requirement for teens who have a license and their friends who ride with them. An adult or older teen should be present for activities like swimming — encourage use of the "buddy system" for swimming. Teach your children basic water safety techniques and how to swim. It could save their lives. "Childproof" your household by covering electrical outlets and keeping all drugs and cleaning products out of the reach of young children. Have the number of your local Drug and Poison Information Center posted near the telephone. Talk with your children and be involved in their lives and schoolwork. One concerned adult in the life of a child can make a tremendous difference in how well a child does. Talk to your children about drugs and teach them about sex before they begin experimenting on their own. The older they get, the more information they need. All information should be age appropriate. Teach them your values. Know who their friends are and what kind of behavior they engage in. Peers have a powerful influence on teens.

www.netwellness.uc.edu 